\title{
Forensic Nanopathology: a New Frontier of Medicine
}

\section{Editorial}

Nanotechnology is an important aspect of the technological progress of the XXI century and has the same societal, scientific and political impact as the discovery of how to exploit electricity had in the XIX century. But everybody knows that there is no technological progress which is free from undesirable side effects, and this represents the other side of the same coin. Nanotoxicology is about part of those side effects. Since the first syntheses of the nano sized entities called nanoparticles (NPs), e.g. fullerene, Carbon nanotubes, quantum dots, etc. and the discovery of their exceptional properties, many scientists has expressed concern about their possible toxicological impact on humans, animals and environment.

At the beginning, the reason of that concern was purely theoretical. The very small size comparable with that of proteins, enzymes, vitamins prompted a suspicion about their not impossible interaction with those natural compounds whose effects were unknown. The concerns were promptly denied by nanotechnologists impressed by the properties of matter at nanoworld scale. At that time, not much longer than 20 years ago, it was already certain that those entities, if inhaled, could cross the lung barrier and, if ingested, could not be wholly eliminated by the feces: the obvious conclusion was that they remained inside the organism [1].

The in-vitro and in-vivo toxicological studies performed later showed controversial results, as they led to opposite results: some scientists (mostly biologists and molecular biologists) believed that nanoparticles are safe since they do not induce an immediate cell death and there is not a clear dose-response answer. Others (mainly bio-engineers, chemists, etc.) replied that they can have a potential to induce biological, maybe pathological, reactions. Now the piece of evidence about NPs being able to cross any physiological barriers, thus implying their dispersion throughout the whole body and a potential risk for cells and cells' content, DNA included, is universally shared. Nevertheless, discrepancies continue.

Those controversial results are due, at least in part, to the physical properties of NPs, particularly to their strong tendency to adhere to each other that makes isolated (i.e. not aggregated) NPs hard to come by. The in-vitro and in-vivo tests use NPs' micron-sized aggregates that change the boundary conditions of the experiments and, as a consequence, distort the interpretation of the scientific results. NPs are very hard to handle and that prevents researchers to carry out true nanotoxicological tests and get reliable results.

Controversies or not, the free entrance of NPs in the organism is now demonstrated in practice by experience and by some applications of Nanomedicine based on that property. New therapies for neurological diseases are based on the use of biodegradable NPs, able to cross the brain-blood barrier, loaded with drugs that, if not properly carried, would be rejected. Genetic

Volume 4 Issue 1 - 2016
Antonietta M Gatti*
Department of Nanodiagnostics, National Council of Research
of Italy, Italy
*Corresponding author: Antonietta M Gatti, Department of
Nanodiagnostics, National Council of Research of Italy, ISTEC
(Faenza), c/o Nanodiagnostics, Modena, Italy, Email:
gatti@unimo.it
Received: July 13, 2016 | Published: July 19, 2016

therapies, for example, use NPs as a Trojan horse to introduce DNA fragments into the cell, something that used to be very hard, if not impossible at all, to accomplish.

Now there is no doubt: NPs enter the body and most of them are distributed throughout its organs via the blood circulation. The question is: how do those tiny foreign bodies interact with the organism's complexity? The so-called nano-biointeraction is the key point to understand how the pathologies caused by NPs are triggered and explain the mechanisms at its roots.

Nanopathology is the branch of learning that studies the interactions of submicron and nano sized foreign bodies with the organism and their possible side effects. Nano-biointeraction and Nanopathology are two sides of the same coin, nanobiointeraction being the micro and Nanopathology the macro aspect of those phenomena [2].

Over the years, we have developed protocols to identify those entities in pathological tissues by means of Scanning Electron Microscopy. Our studies allowed to demonstrate that NPs are free to negotiate virtually any human physiological barrier, and that their concentration in tissues and organs affects the onset of a disease, the speed at which the phenomenon occurs and its seriousness.

The Nanopathological studies on tissues affected by lymphoma, leukemia, and other forms of cancer and sometimes by mysterious or rare diseases like cryoglobulinemia verified the presence of sub-micro and nanosized foreign bodies. Sometimes those NPs are incidental, i.e. produced unintentionally, as side effects, by high-temperature combustion/explosion processes, or are engineered, i.e. made on purpose. The identification of their chemical composition by means of the X-ray microprobe of an Energy Dispersive System allows to understand what type of exposure the patient underwent and to identify the source of pollution. This piece of information can allow to trace the contamination in the environment where the patient lived or worked and, when possible, to eliminate it. 
This aspect used little or not at all as it is in present forensics, has a great potential. In fact, the study of the body's internal contaminations, their identification in the environment and their chemical overlapping allow to understand the link among the source, the exposure and the pathology, a link essential in claims where it is mandatory to demonstrate that a correlation exists. Claims and reimbursement in working places but also in the surroundings of contaminating factories are sustainable only through objective demonstration. In the case of nanotechnological factories, contamination can interest not only the NPs fabricated there and the entire fabrication process, but also the interactions of those NPs with the environment and their possible modification. In some cases, it is important to be aware of all the production steps to be able to correlate pathologies among the workers and working-place or working-process pollution.

If the characterization of a bullet in the body of a murdered person can put on the right track to identify the gun and, hopefully, the gun's owner/killer, in the same way, detecting and characterizing the NPs (called "invisible bullets") in a pathological tissues can allow pinpointing their source and it's responsible.

The study of more than 3,000 pathological tissues affected by pathologies of unknown etiology, all with the presence of submicron- and nano-sized foreign bodies, convinced us of the forensic value of these analyses. Our cases involve also a remarkable number of soldiers who acted in war fields. In the cases of the soldiers who served in the I and the II Gulf War or in the Balkan War, we found nanopollution produced by hightechnology ammunition [3]. Journalists who first noted the increase of the illnesses and death rate of the soldiers after the end of the war missions put forward the hypothesis of a relationship between the use of slightly radioactive DepletedUranium weapons and the pathologies, in some cases fatal. Our studies verified the total absence of Uranium contamination in those tissues, but an important presence of sub-micron and NPs, coming from the blast's effect. Their composition varied from stainless steel to zirconia, from Titania to very particular alloys. Unknown-before particle compositions are the result of the melting of the bomb with the target and part of the environment. The high temperature breaks the original molecules into much smaller ones or, often, into atoms and those molecules and atoms aggregate randomly forming polluting particles that can't be found in a "normal", e.g. urban, contamination.

Studying a civilian patient died of lymphoma at the hospital of Sarajevo when the Balkan War was already over; we found the deepest and finest contamination of his lymph nodes by $10 \mathrm{~nm}$ - sized particles of Carbon-Lead-Chlorine-Bromine. Such a small size, morphology (spherical) and chemical composition induced us to hypothesize an exposure to an important source of hightemperature-produced soot. As a matter of fact, the town of Pancevo, where that subject came from, was home to the largest oil refinery of the country, and that plant had been bombed for three days by the NATO Army. An 800 m-high column of sooty fumes was formed, polluting the area for months. The patient inhaled and most probably ingested that soot along with contaminated vegetables and fruit. Then he moved to Sarajevo where he grew ill and eventually died because of the exposure he had undergone elsewhere.

In another case, this time regarding a British woman affected by so-called "cryptogenic" liver granulomatosis, we identified 50nm-sized Gold NPs that an accurate anamnestic investigation revealed coming from colloidal-Gold injections administered long ago in a knee to cure her osteoarthritis. The result was a relief from the pains she suffered but a slow dispersion throughout the body of the NPs with the liver as main target. In that case, a foreign-body granulomatosis eventually transformed into a granulomatosis had been the consequence.

That kind of experience proves very useful in case of patients who were treated with non biodegradable NPs either for therapeutic or for diagnostic (e.g. imaging) purposes and then developed a pathology. In most instances, a correlation can be demonstrated between administration and pathology contracted as side-effect. Also workers employed in nanotechnology laboratories can often prove that their illness was triggered by the NPs they worked with and that contaminated the place.

Forensic Nanopathology is a novel discipline that, if properly understood and used, can help Medicine to resolve court cases and, in addition, to understand better the mechanism at the base of many diseases we are still wondering about.

\section{References}

1. Nemmar A, Hoet PH, Vanquickenborne B, Dinsdale D, Thomeer M, et al. (2002) Passage of inhaled particles into the blood circulation in humans. Circulation 105(4): 411-414.

2. Gatti AM, Montanari S (2008) Nanopathology, The Health Impact of Nanoparticles, PanStanford Pub. Singapore 1-291.

3. Gatti AM, Montanari S (2015) Case Studies in Nanotoxicology and Particle Toxicology, Elsevier, USA, p. 1-276. 